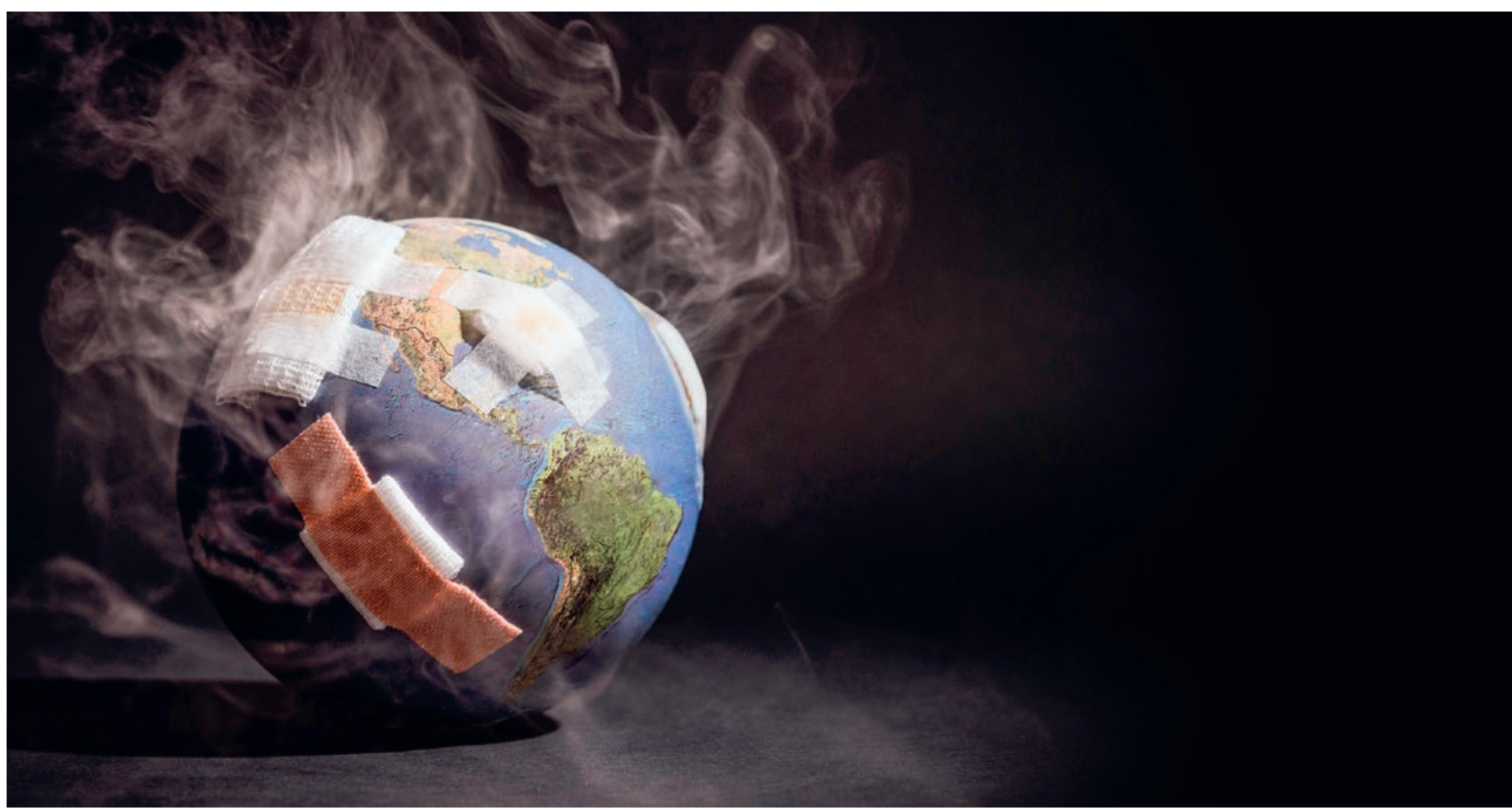

Die Erde leidet: Grosse Investitionen in fossile Energien schaden dem Klima.

\title{
Die Klimakatastrophe und unsere Pensionskassen
}

\section{René Jaccard ${ }^{a}$, Ueli Hagnauer}

${ }^{a}$ Dr. med., Wissenschaftskommission Klima-Grosseltern; ${ }^{b}$ Dr. med., Koordinator BE, Klima-Grosseltern

Wenn die Bemühungen um Erholung von den Folgen der Covid-19-Pandemie nicht postwendend in die nächste Katastrophe, jene der ungebremsten Klimakrise, führen sollen, müssen Kriterien der Nachhaltigkeit den Pandemie- und Postpandemie-Massnahmenkatalog bestimmen (Stichwort Green recovery) [3].

Mitten in die zweite Welle der Corona-Pandemie kommt die Meldung: Die Zahl der Menschen, die direkt durch Klimaerwärmung und den ihr zugrunde liegenden Verbrauch fossiler Brennstoffe sterben, steigt weiterhin Jahr für Jahr (2019 weltweit: 7,5 Mio.). Klimabedingte Herz-, Lungen- und Nierenerkrankungen nehmen stetig zu, und das Gleiche gilt für Arbeitsausfälle infolge der Klimaerwärmung (2019: weltweit 302 Mia. Stunden) [1]. Parallel dazu haben sich die relevanten Klima-Messgrössen weiterhin verschlechtert: Im Vergleich zu 1950 hat die Jahrestemperatur global um $1,2^{\circ} \mathrm{C}$, in der Schweiz um $1,7^{\circ} \mathrm{C}$ zugenommen. Die globale Konzentration des Haupt-Treibhausgases $\mathrm{CO}_{2}$ ist von 250 auf aktuell 410 ppm angestiegen [2].

\section{Was können wir tun?}

Bemühungen um eine bessere Bekämpfung von gesundheitlichen Folgen von Hitzetagen haben in der Schweiz bereits Erfolg gezeigt [4]; schweizweite Projekte zur Erhebung der durch das Gesundheitswesen verursachten Treibhausgasemissionen sind angelaufen, dies mit dem deklarierten Ziel ihrer Reduktion (Stichwort: Green Hospitals) [5]; die Ärztinnen und Ärzte für Umweltschutz haben schon vor einiger Zeit einen Leitfaden zur Reduktion des $\mathrm{CO}_{2}$-Fussabdruckes in der Praxis publiziert [6].

Hier möchten wir auf eine weitere Möglichkeit mit besonders grossem Wirkungspotenzial eingehen: Es geht 
darum, wie die gesetzlich vorgeschriebenen Pensionskassen-Spargelder angelegt werden.

\section{Investitionen sind klimarelevant}

Die Gesamtsumme der in der Schweiz obligatorisch angesparten Vorsorgegelder beträgt rund CHF 1000 Mia. (entspricht ca. $1 / 7$ der Gesamtsumme des Finanzplatzes Schweiz). Die gegenwärtige Anlagepraxis der Pensionskassen führt auf einen Temperaturanstieg von $4-6^{\circ} \mathrm{C}$ bis zum Ende des Jahrhunderts hin, dies insbesondere aufgrund hoher Investitionen in fossile Energien, inkl. der besonders klimaschädlichen und ungesunden Kohle [7]. Von den mindestens 300000 in der Schweiz im Gesundheitssektor Tätigen ist ein Teil in staatlichen Institutionen angestellt und automatisch entsprechenden Kassen zugeteilt - ein grosser Teil ist aber in Kleinunternehmen versichert, die wiederum in der Wahl ihrer Pensionskasse frei sind.

Kürzlich ist ein Rating von 110 Schweizer Pensionskassen veröffentlicht worden [8]: Aktuell weisen $8 \%$ ein auf Nachhaltigkeit und Klimaschutz ausgerichtetes Portfolio auf. Zu den schlecht klassierten (55\%!) gehören leider drei wichtige Pensionskassen, die vorwiegend im Gesundheitswesen von der privaten Ärzteschaft genutzt werden: «PAT», «Pro Medico» und «VSAO» [8].

\section{Gute Performance nachhaltiger Investitionen}

Global wurde seit 2008 vonseiten des Gesundheitssektors ein Gesamtbetrag von USD 42 Mia. divestiert (Abzug von Investitionen aus den fossilen Energien), woran sich 23 ärztliche Organisationen aus Grossbritannien, Kanada, Australien und Deutschland beteiligten [1]. Es ist nicht einzusehen, warum nicht auch in der Schweiz mit ihrer schlagkräftigen Standesorganisation FMH unsere Pensionskassen verbindlich auf eine Anlagepolitik verpflichtet werden, die mit den Klimazielen des Pariser Abkommens von 2015 (COP 21) übereinstimmt. Heute noch Investitionen in fossile Energien zu tätigen heisst nicht nur, der sich entwickelnden Klimakrise freien Lauf zu lassen und die Gesundheit vulnerabler Teile der Bevölkerung sowie künftiger Generationen zu untergraben [1]. Es bedeutet auch, grosse Verluste unserer Ersparnisse zu riskieren. Zur kraftvollen Vertretung unserer Interessen gegenüber den Pensionskassen können neben der FMH auch wir alle beitragen: Beziehen wir uns auf das Klimaverträglichkeitsranking unserer Pensionskasse, und fragen wir, was die Pläne zur Verbesserung sind, dann haken wir nach, wenn wir mit dem Bescheid nicht zufrieden sind: Entweder verpflichtet sich unsere Kasse aufs Umschwenken zu einem mit Klimaschutz vereinbaren Kurs, oder wir wechseln ganz einfach auf eine der bereits heute klimafreundlich agierenden Pensionskassen (die übrigens durchaus gute Performances aufweisen). Denn Klimaschutz ist nicht zuletzt Gesundheitsförderung!

\section{Bildnachweis}

도 Myboys.me | Dreamstime.com, Symbolbild

\section{Literatur}

1 Watts N, Amann M, Arnell N, et al. The 2020 report of the Lancet Countdown on health and climate change: responding to converging crises; Published online December 2, 2020: https://doi.org/10.1016/S0140-6736(20)32290-X

2 BAFU, et al. 2020: Klimawandel in der Schweiz. Indikatoren zu Ursachen, Auswirkungen, Massnahmen. Umwelt-Zustand Nr. 2013, 105 Seiten.

3 EU, July 3, 2020: Boosting the EU's green recovery, online: https:// ec.europa.eu/commission/presscorner/detail/en/IP_20_1250

4 Ragettli MS, Röösli M. Gesundheitliche Auswirkungen von Hitze in der Schweiz und die Bedeutung von Präventionsmassnahmen. Swiss TPH, Juli 2020.

5 Muir K, Keller R, Stucki M. Bringing green best practice into hospitals with an LCA approach. July 29th 2020, Discussion Forum Nr. 74, IUNR u. ZHAW.

6 AefU-Ratgeber Ökologie in der Arztpraxis, online http://www. aefu.ch/fileadmin/user_upload/aefu-data/b_documents/themen/ praxisoekologie/Ratgeber_Energiecheck.pdf

7 BafU u. PACTA, October 2017: Quantifying the alignment of Swiss pension funds and insurances with the Paris agreement, online https://www.bafu.admin.ch/bafu/de/home/themen/klima/ fachinformationen/klima-und-finanzmarkt.html

8 Klima-Allianz Schweiz, 2. November 2020: Erst ein Bruchteil der Pensionskassen investiert klimaverträglich. Online https://www. klima-allianz.ch/klima-rating/?utm

source $=$ Newsletter+Finanzwelt-Politik $+\mathrm{DE}+200702 \& u$ tm campaign=b6ab87ae03-EMAIL_CAMPAIGN_2018_08_30_08_31_ COPY_O1\&utm_medium $=$ email $\& u$ tm_term $=0$ f 4 fff $8 \bar{b} 12 \mathrm{~b} 2-\mathrm{b} \overline{6} \mathrm{a}-$ b87ae03-587539512

\section{Das Wichtigste in Kürze}

- Die Klimaerwärmung bedroht nicht nur die Umwelt, sondern auch klimabedingte Erkrankungen nehmen stetig zu. Klimaschutz ist also auch Gesundheitsförderung.

- Die Anlage der gesetzlich vorgeschriebenen Spargelder in Pensionskassen mit nachhaltigem und auf Klimaschutz ausgerichtetem Portfolio hat grosses Wirkungspotenzial: die Gesamtsumme der angesparten Vorsorgegelder beträgt in der Schweiz $1 / 7$ der Gesamtsumme des Finanzplatzes.

- Entweder verpflichtet sich unsere Kasse auf einen nachhaltigen Kurs, oder wir wechseln ganz einfach auf eine der bereits heute klimafreundlich agierenden Pensionskassen. 